\title{
Multiple transmit antennas for low PAPR spatial modulation in SC-FDMA: single vs. multiple streams
}

\author{
Darko Sinanović ${ }^{*} \mathbb{D}$, Gordan Šišul ${ }^{2}$, Adrian Satja Kurdija ${ }^{2}$ and Željko $\|_{i c ́}{ }^{2}$
}

\begin{abstract}
The main reason for the selection of single-carrier frequency division multiple access (SC-FDMA) modulation technique in mobile high data rate communications lays in its low value of peak-to-average power ratio (PAPR). If spatial modulation, a multiple-input multiple-output technique that achieves bitrate increase, is applied to the SCFDMA system in its conventional form, the PAPR level is increased. Hence, based on previously mentioned, in this paper, we provide the general design criteria for low PAPR spatial modulation (LPSM) implemented in SC-FDMA systems with multiple transmit antennas. The straightforward generalization of LPSM is derived and it is shown that it requires the number of transmit antennas to be doubled in order to add another bit in the spatial component. Based on that, the alternative generalized technique with higher number of spatial bits is proposed. As these techniques present single-stream generalizations, the approach with multiple-streams is proposed as well. After PAPR and performance comparisons, it is concluded that single-stream techniques outperform multiple-stream techniques in the cases of low overall data rate, whereas multiple-stream approach is better in high overall data rate scenarios. All proposed techniques maintain low PAPR level on all transmit antennas.
\end{abstract}

Keywords: Spatial modulation, SC-FDMA, PAPR, MIMO

\section{Introduction}

Spatial modulation (SM) has been introduced recently as an attractive multiple-input multiple-output (MIMO) technique that achieves bitrate increase $[1,2]$. It has been initially proposed for the conventional quadrature amplitude modulation (QAM) or phase shift keying (PSK) single-carrier modulations in flat fading channels [1]. In SM, each QAM/PSK symbol is transmitted via only one antenna and the receiver detects the QAM/ PSK symbol and the active transmit antenna. Therefore, the index of transmit antenna is used for the transmission of the additional bits. The selection of the active antenna is performed after the amplifier and the transmitter has only one radiofrequency (RF) chain. As the narrow subchannels in orthogonal frequency division multiplexing (OFDM) can be considered as flat fading channels, the migration to OFDM is straightforward and has been given in [2]. The benefit of low complexity

\footnotetext{
* Correspondence: d.sinanovic@nosbih.ba

${ }^{1} \mathrm{NOSBIH}$, Sarajevo, Bosnia and Herzegovina

Full list of author information is available at the end of the article
}

transmitter is lost in OFDM and the transmitter requires the number of RF chains to be equal to the number of transmit antennas [3]. Hence, the main benefit of SM in OFDM is in performance gain [2].

The generalization of SM that uses multiple active transmit antennas, regarded as a generalized SM (GSM), has been studied in the literature [4-8]. In GSM, the transmitter activates the subset of transmit antennas. In general, the two approaches have been used. In the first approach $[4,5]$, regarded as a single-stream GSM, only one QAM/PSK sequence is created and each QAM/PSK symbol is transmitted via the selected subset of transmit antennas. Hence, all active antennas transmit the same QAM/PSK symbol. In the other approach [6-8], regarded as a multiple-stream GSM, multiple QAM/PSK sequences are generated and each sequence is transmitted via one of the active antennas. There are as many QAM/PSK sequences as there are antennas in the subset. Thereby, in one symbol period, multiple QAM/PSK symbols are transmitted via multiple active antennas belonging to the subset, one symbol per antenna, and the 
other antennas are inactive. Multiple-stream GSM can be regarded as a combination of SM and spatial multiplexing. A detailed analysis of GSM is given in [9].

SC-FDMA is an OFDM-based modulation and multiple access technique characterized by the low value of peak-to-average power ratio (PAPR) of output signal and the performance similar to OFDM [10]. Hence, it is suitable for the implementation in the uplink of mobile communications and has been selected in long-term evolution (LTE) standard [11]. In order to be suitable for practical usage, any MIMO technique implemented in SC-FDMA has to maintain low PAPR level on all transmit antennas. The issue of PAPR in SC-FDMA has been studied recently in [12-15].

Recently, in [16], it has been shown that the conventional SM is not suitable for SC-FDMA as it increases PAPR and low PAPR SM (LPSM) has been proposed. The cases of two and four transmit antennas have been studied and it has been shown that LPSM shows good performances in different channel models for different receivers while maintaining low PAPR level on all transmit antennas. As it is the case for SM in OFDM, LPSM in SC-FDMA requires as many RF chains as transmit antennas. In the conventional SM, the output signal can be presented as a multiplication of the QAM/PSK symbol with a vector consisting one for the active antenna and zeros for all the others. The additional information is placed in the index of the active antenna. However, in LPSM, the output signal is generated by a multiplication of the QAM/PSK symbol with a vector consisting complex coefficients of the equal amplitude levels and predefined phases for all antennas. Therefore, output signal is obtained as phase shifted QAM/PSK symbol normalized in order to maintain the same output power level. The equal amplitude level preserves the PAPR level of SCFDMA signal on all antennas, whereas phase shifts introduced with the multiplication are used for conveying the additional bits in the spatial component. The receiver extracts the bits from the spatial component by determining all phase shifts from all transmit antennas. Therefore, LPSM can be considered as a coding performed as a multiplication of QAM/PSK symbols with the corresponding vector of LPSM coding coefficients. However, it is not a space-time coding technique as it does not provide diversity.

To the best of our knowledge, the generalization of SM in SC-FDMA has not been studied. As the conventional SM increases PAPR, in this paper we studied LPSM further, observing the cases with multiple transmit antennas, i.e., more than four, to create an acceptable generalized SM for SC-FDMA. The main constraint is that the PAPR level has to be preserved. As it has been shown in [16], the low PAPR condition in SC-FDMA requires that all transmit antennas are active all the time. Therefore, the conclusions from GSM cannot be applied in the same manner to LPSM as a subset of active transmit antennas cannot be used. However, the approaches of single and multiple streams can be considered and adapted to LPSM.

The contributions of this paper are the generalizations of LPSM for the cases of multiple transmit antennas with single and multiple streams. In the single-stream generalized LPSM (SS-GLPSM), a single QAM/PSK sequence is generated and generalized LPSM coding is performed over all transmit antennas and the proof for optimal coefficients selection is given. This can be observed as a straightforward generalization of LPSM for the case of more than four transmit antennas. In this case, as in the conventional SM, the number of bits conveyed in the spatial component grows logarithmically with the number of antennas and in order to add another bit in the spatial component, the number of transmit antennas must be doubled. Therefore, in addition to SS-GLSPM, we propose generalized SS-GLPSM (GSS-GLPSM) that places more bits in the spatial component for the same number of transmit antennas to overcome this limitation.

Alternative approach to increase the number of overall bits is proposed as multiple-stream generalized LPSM (MS-GLPSM) where multiple QAM/PSK sequences are generated and each sequence is SS-GLPSM-coded over the subset of transmit antennas and transmitted via the defined subset. The union of all subsets spans over all transmit antennas. This can be seen as a combination of LPSM and spatial multiplexing. The main challenge of this approach is the interference between the streams, hence more complex receivers with interference cancelation should be used.

Hence, the novelties of this paper are as follows:

- The straightforward generalization of LPSM [16] to the case of multiple transmit antennas, denoted as SS-GLPSM, and the proof for the optimal coding coefficients selection;

- The alternative generalization of LPSM that places more bits in the spatial component, denoted as GSSGLPSM;

- The combination of LPSM and spatial multiplexing, denoted as MS-GLPSM.

It is worth noting that, in this paper, GLPSM is considered as an open-loop MIMO technique and the transmitter does not have the information about the conditions in the channels. Furthermore, GLPSM maintains the PAPR level of the conventional SC-FDMA signal, hence it is not an PAPR reduction technique [17-19].

The paper is organized as follows. Section 2 of this paper provides the system model and SS-GLPSM with optimal coefficients is presented. GSS-GLPSM, an alternative of SS-GLPSM that conveys more bits in the 
spatial component, is presented in Section 3. The approach with multiple streams, denoted as MS-GLPSM, is presented in Section 4. Any MIMO technique, aimed to be used in SC-FDMA, has to maintain the PAPR level at the acceptable level, therefore in Section 5, that provides the results and discussion, PAPR comparison is given prior to the performance comparison. The paper is concluded in Section 6. The mathematical proof for optimal coefficients selection and the table of coefficients for eight transmit antennas are given in Appendix $\mathrm{A}$ and Appendix B in Section 7.

Notation: italic symbols denote scalar values and bold symbols denote vectors/matrices; $(.)^{\mathrm{H}}$ is Hermitian vector/ matrix; $\mathcal{I}_{M}$ denotes $M \times M$ identity matrix; $j$ is imaginary unit; $\|\cdot\|_{\mathrm{F}}$ denotes Frobenius norm of a vector/matrix; $\mathrm{E}\{\cdot\}$ denotes the expected values; and $\operatorname{Pr}(\cdot)$ denotes the probability.

\section{Single Stream GLPSM}

\subsection{System model}

As SC-FDMA is an OFDM-based technique, GLPSM requires as many RF chains as the transmit antennas, i.e., $N_{T}$ [16]. Hence, there are $N_{T}$ output amplifiers and all transmit antennas are simultaneously active. In analogy to [16], GLPSM coding in SC-FDMA is implemented as a multiplication of QAM/PSK symbol with the corresponding coding coefficients for all transmit antennas. Unlike the conventional SM, all QAM/PSK symbols are transmitted via all transmit antennas simultaneously and the additional spatial bits are not in the index of the active transmit antenna, but in the coding coefficients of all antennas.
The transmitter block scheme for the case of $N_{T}$ transmit antennas is presented in Fig. 1.

It is assumed that the user occupies $M$ out of $N$ available subcarriers and the underlying modulation is QAM (or PSK) with the constellation size $2^{L}$. Let $K$ denote the number of bits to be placed in the spatial component. Input bit sequence, consisting of $M(L+K)$ bits, is divided into two groups. The first group of $M L$ bits is mapped to $M$ QAM/ PSK symbols sequence $\left\{s^{(i)}\right\}_{i=1}^{M}$, and the remaining $M K$ bits, $\left\{b_{1}^{(i)}, b_{2}^{(i)}, \ldots, b_{K}^{(i)}\right\}_{i=1}^{M}$, are used for GLPSM coding. As in the conventional SM, the GLPSM coding is performed per QAM/PSK symbol, hence the subsequences of $L$ and $K$ bits are used for the coding in a way that $L$ bits are used for QAM/PSK modulation and $K$ bits are used for GLPSM coding for the corresponding QAM/PSK symbol. GLPSM coding, as presented in Fig. 1, is performed as a multiplication of QAM/PSK symbol and a spatial symbol. Here, we define the spatial symbol in analogy to the transmit antenna index in the conventional SM, as a vector of complex coding coefficients that is used for conveying bits in the spatial component. Therefore, $K$ bits are used for the selection of the spatial symbol and GLPSM coding is performed. For each transmit antenna $t, t=1,2, \ldots, N_{T}$, GLPSM coding is performed by multiplying the QAM/ PSK symbol with the corresponding coding coefficient placed on tth position in the spatial symbol vector.

As in the conventional SM, the number of transmit antennas is equal to the number of spatial symbols, i.e., $N_{T}=2^{K}$, therefore, a spatial symbol carries $K$ bits. Hence, if $K$ bits are mapped to $p$, where $p=0,1, \ldots, N_{T}-1$, then the spatial symbol can be written as a vector $W_{p}=\left[a_{1, p}\right.$,

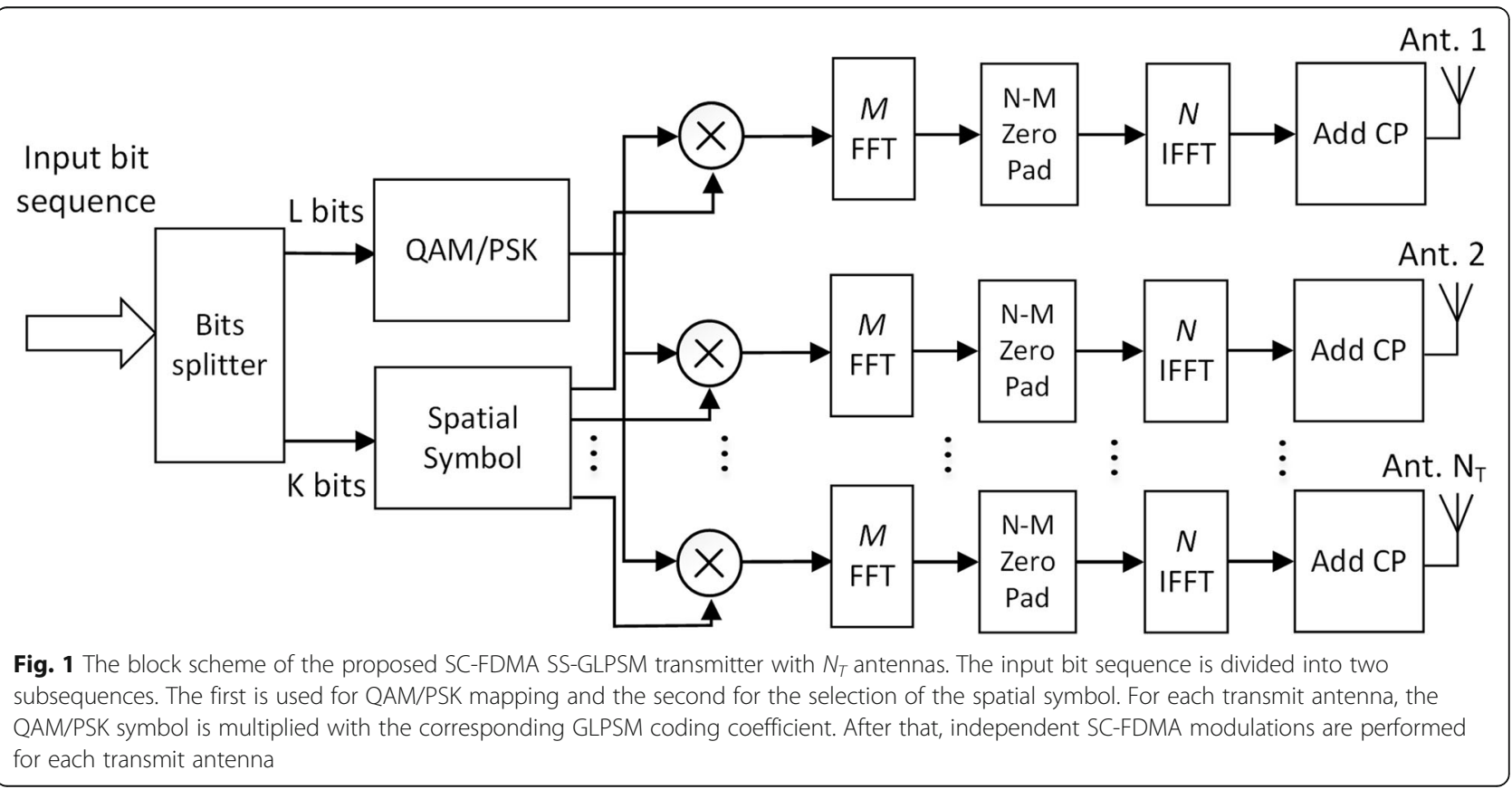


$\left.a_{2, p}, \ldots, a_{N_{T}, p}\right]$. This implies that $K$ bits can be considered as spatial bits.

Further, if we define the GLPSM symbol as a product of the QAM/PSK symbol $s$ and the spatial symbol $W_{p}$, then the GLPSM symbol can be written as $\boldsymbol{v}_{p, s}=s \boldsymbol{W}_{p}$. Hence, the GLPSM symbol is a vector of length $N_{T}$, consisting of products of a QAM/PSK symbol $s$ and the spatial symbols coefficients, as presented in Fig. 1.

If we denote $L$ the number of bits transmitted via QAM/PSK symbol then the constellation size of QAM/ PSK modulation is $2^{L}$ and as the number of spatial symbols is $2^{K}$, there are $2^{L+K}$ possible GLPSM symbols. This implies that the number of bits per subcarrier in one SC-FDMA symbols is increased from $L$ to $L+K$.

After GLPSM coding, SC-FDMA modulation is performed. For all $N_{T}$ transmit antennas, each block of $M$ outputs of the multiplications is transformed to the frequency domain using $M$-point FFT (fast Fourier transform). Prior to the $N$-point inverse fast Fourier transform (IFFT) operation, $N-M$ zeros are padded for the unused subcarriers. The outputs of IFFT operations are blocks of $N$ samples in the time domain. After the cyclic prefix insertion, the output signals are amplified and transmitted via each transmit antenna. It can be concluded that with GLPSM, one SC-FDMA symbol transmits $M(L+K)$ bits, providing an increase of $M K$ bits per SC-FDMA symbol. It is worth noting that one SC-FDMA symbol transmits $M$ GLPSM symbols.

Different receivers for SS-GLPSM can be derived directly from the receivers given in [16]. However, for the sake of simplicity, in this paper only linear minimum mean-square error (MMSE) receiver [16] is considered. After the channel state estimation, the receiver equalizes the signal using MMSE and detects the GLPSM symbol per subcarrier. As there are $2^{L+K}$ possible GLPSM symbols, the receiver finds the nearest GLPSM symbol and makes the decision of $L+K$ bits.

\subsection{Coding coefficients selection}

After the GLPSM coding scheme is presented, the optimal values for the coding coefficients have to be found. If $K$ bits are mapped to $p$, then the transmitter transmits the corresponding QAM/PSK symbol over all transmit antennas, multiplying it with the coefficients $a_{t, p}$ for each transmit antenna $t\left(t=1,2, \ldots, N_{T}\right)$. Hence, there are $N_{T}^{2}$ coefficients to find.

As mentioned, GLPSM is considered in this paper as an open-loop MIMO technique, i.e., the transmitter does not have the channel state information. Therefore, the criteria for the selection of optimal values for the coefficients $a_{t, p}$ are the maximization of the minimum distance between any two possible GLPSM symbols. This is similar to the approach in [20] where closed-loop SM is considered and the minimum distance is maximized at the receiver side. However, in this paper open-loop MIMO is considered and the minimum distance is maximized at the transmitter side.

Using the same approach as in [16], it can be easily shown that in order to preserve low PAPR of all output signals and to avoid amplitude fluctuations, all coefficients must have the same absolute value. Assuming unitary total output power, it can be shown that for all $a_{t, p},\left|a_{t, p}\right|=\sqrt{1 / N_{T}}$ has to be satisfied. It is hard to obtain PAPR analytically [4], therefore PAPR is analyzed in Section 6 via simulation outcomes and it is confirmed that the low PAPR level is preserved on all transmit antennas.

Therefore, we have

$$
a_{t, p}=\sqrt{\frac{1}{N_{T}}} e^{j \phi_{t, p}}
$$

where $\phi_{t, p}$ are the phases of the corresponding coding coefficients and the optimal values for the phases have to be found.

As there are many different QAM/PSK modulations, there is no general expression for the scalar $s$ that presents QAM/PSK symbol. However, for all common modulations, it can be assumed that the constellations are symmetric with respect to the origin and hence, for every QAM/PSK symbol $s$, there is also a symbol $-s$.

In order to find the optimal values for the coefficients, three cases can be distinguished.

1) The distance between two GLPSM symbols when their spatial symbols are equal.

Let $s_{i}$ and $s_{k}$ denote two different QAM/PSK symbols. The squared distance between any two GLPSM symbols is

$$
D_{(1)}^{2}=\left|s_{i} \boldsymbol{W}_{p}-s_{k} \boldsymbol{W}_{p}\right|^{2}=\left|s_{i}-s_{k}\right|^{2}\left|\boldsymbol{W}_{p}\right|^{2}
$$

As all coding coefficients have the same absolute value, the distance does not depend on the phases.

2) The distance between two GLPSM symbols when their spatial symbols are different and QAM/PSK symbols have the same absolute value.

Let $p \neq q\left(p, q=0,1, \ldots, N_{T}-1\right)$, defining different spatial symbols $W_{p}$ and $W_{q}$, and let $s_{i}$ and $s_{k}$ denote two, not necessarily different, QAM/PSK symbols of the same absolute value. In that case, $s_{k}$ can be written as $s_{k}=s_{i} e^{j \alpha}$ and the squared distance between the GLPSM symbols is 


$$
D_{(2)}^{2}=\left|s_{i} \boldsymbol{W}_{p}-s_{k} \boldsymbol{W}_{q}\right|^{2}=\left|s_{i}\right|^{2}\left|\boldsymbol{W}_{p}-e^{j \alpha} \boldsymbol{W}_{q}\right|^{2} .
$$

As there are many different QAM/PSK modulations that can be used, $\alpha$ can have different values.

The spatial symbols $W_{p}$ consist of $N_{T}$ complex coding coefficients of the equal absolute values. Therefore, they can be written as vectors of length $2 N_{T}$ consisting of real values

$$
W_{p}=\sqrt{\frac{1}{N_{T}}}\left[\cos \phi_{1, p}, \sin \phi_{1, p}, \ldots, \cos \phi_{N_{T}, p}, \sin \phi_{N_{T}, p}\right] .
$$

Further follows

$$
\begin{aligned}
\left|W_{p}-W_{q} e^{j \alpha}\right|^{2}= & \frac{1}{N_{T}} \sum_{t=1}^{N_{T}}\left(\left(\cos \phi_{t, q}-\cos \left(\phi_{t, q}+\alpha\right)\right)^{2}\right. \\
+ & \left.\left(\sin \phi_{t, p}-\sin \left(\phi_{t, q}+\alpha\right)\right)^{2}\right)(\underline{5}) \frac{2}{N_{T}} \sum_{t=1}^{N_{T}} \\
& \left(1-\cos \left(\phi_{t, p}-\phi_{t, q}-\alpha\right)\right) .
\end{aligned}
$$

In order to maximize the distance, the value $A$ defined as

$$
A=\sum_{t=1}^{N_{T}} \cos \left(\phi_{t, p}-\phi_{t, q}-\alpha\right)
$$

has to be minimized. Eq. (6) can be written as

$$
A=\cos \alpha \sum_{t=1}^{N_{T}} \cos \left(\phi_{t, p}-\phi_{t, q}\right)+\sin \alpha \sum_{t=1}^{N_{T}} \sin \left(\phi_{t, p}-\phi_{t, q}\right)
$$

As the phase difference between $s_{i}$ and $s_{k}$ is $\alpha$, the difference between $s_{i}$ and $-s_{k}$ is $\alpha+\pi$. Here, we use the existence of a QAM/PSK symbol symmetric with respect to the origin. Therefore, for the distance between $s_{i}$ and $-s_{k}$, we have to minimize the value $B$ defined as

$$
B=-\cos \alpha \sum_{t=1}^{N_{T}} \cos \left(\phi_{t, p}-\phi_{t, q}\right)-\sin \alpha \sum_{t=1}^{N_{T}} \sin \left(\phi_{t, p}-\phi_{t, q}\right) .
$$

Minimizing (7) and (8) for various $\alpha$ implies that

$$
\sum_{t=1}^{N_{T}} \cos \left(\phi_{t, p}-\phi_{t, q}\right)=\sum_{t=1}^{N_{T}} \sin \left(\phi_{t, p}-\phi_{t, q}\right)=0
$$

has to be satisfied. Further, it can be concluded that the phase difference between any two spatial symbols, rather than the actual phases, is relevant. From (9), it can be easily shown that for any phase shift $\beta$,

$$
\sum_{t=1}^{N_{T}} \cos \left(\phi_{t, p}-\phi_{t, q}+\beta\right)=\sum_{t=1}^{N_{T}} \sin \left(\phi_{t, p}-\phi_{t, q}+\beta\right)=0
$$

This implies that rotating all phases of one spatial symbol by any phase shift does not affect its distances from other spatial symbols. Hence, without loss of generality, it can be assumed that the phases of the first antenna for all spatial symbols are equal and $\phi_{1, p}=0$.

Then, the spatial symbols can be written as

$$
\boldsymbol{W}_{p}=\sqrt{\frac{1}{N_{T}}}\left[1,0, \cos \phi_{2, p}, \sin \phi_{2, p}, \ldots, \cos \phi_{N_{T}, p}, \sin \phi_{N_{T}, p}\right]
$$

As the first two components are irrelevant for the distances, the new vectors, consisting of $2\left(N_{T}-1\right)$ real values, can be created:

$$
W_{p}^{\left[2\left(N_{T}-1\right)\right]}=\sqrt{\frac{1}{N_{T}}}\left[\cos \phi_{2, p}, \sin \phi_{2, p}, \ldots, \cos \phi_{N T, p}, \sin \phi_{N_{T}, p}\right] .
$$

These $N_{T}$ vectors are points on the sphere of radius $\sqrt{\frac{N_{T}-1}{N_{T}}}$ in the $2\left(N_{T}-1\right)$ dimensional space centered in the origin. As the number of transmit antennas is always greater than 1, the number of vectors, i.e., points, is lesser than the number of dimensions.

It is proved in Appendix A that the minimum distance between any two points is maximized when the points form a regular simplex. When the phases are selected as:

$$
\phi_{t, p}=\frac{2 \pi(t-1) p}{N_{T}}
$$

Then vectors given in (12) are vertices of a regular simplex with the centroid in the origin.

3) The distance between two GLPSM symbols when their spatial symbols are different and QAM/PSK symbols do not have the same absolute value.

Let $p \neq q\left(p, q=0,1, \ldots, N_{T}-1\right)$, defining different spatial symbols $\boldsymbol{W}_{p}$ and $\boldsymbol{W}_{q}$, and let $s_{i}$ and $s_{k}$ denote two QAM/PSK symbols of different absolute values. Without loss of generality, $s_{k}$ can be written as $s_{k}=s_{i} m e^{j \alpha}$ where $\alpha$ is the phase shift and $m$ is a real-valued constant, $m>1$. Then, the distance between the GLPSM symbols is

$$
D_{(3)}^{2}=\left|s_{i} \boldsymbol{W}_{p}-s_{k} \boldsymbol{W}_{q}\right|^{2}=\left|s_{i}\right|^{2}\left|\boldsymbol{W}_{p}-m e^{j \alpha} \boldsymbol{W}_{q}\right|^{2}
$$

Further, let $s_{l}$ denote a symbol of the same absolute value as $s_{i}$. This symbol always exists because there is at least the symbol symmetric with the respect to the origin. If the phase difference between $s_{i}$ and $s_{l}$ is $\gamma$, then 
the distance between them for the same spatial symbols equals

$$
D_{(2)}^{2}=\left|s_{i} \boldsymbol{W}_{p}-s_{l} \boldsymbol{W}_{q}\right|^{2}=\left|s_{i}\right|^{2}\left|\boldsymbol{W}_{p}-e^{j \gamma} \boldsymbol{W}_{q}\right|^{2} .
$$

QAM/PSK symbols $s_{i}$ and $s_{l}$ have the same absolute value, as in the case (2), hence the phase shift for any spatial symbol can be introduced without affecting the distances, as shown in (10). After introducing a phase shift $\alpha-\gamma$ to $D_{(2)}^{2}$ follows

$$
D_{(2)}^{2}=\left|s_{i}\right|^{2}\left|\boldsymbol{W}_{p}-e^{j \alpha} \boldsymbol{W}_{q}\right|^{2} .
$$

If the angle between $W_{p}$ and $e^{j \alpha} W_{q}$ is named $\delta$, then from (14) and (16) we have

$$
\begin{aligned}
\frac{D_{(3)}^{2}}{\left|s_{i}\right|^{2}} & =\left|\boldsymbol{W}_{p}-m e^{j \alpha} \boldsymbol{W}_{q}\right|^{2}=\left|\boldsymbol{W}_{p}\right|^{2} \\
& +\left|m e^{j \alpha} \boldsymbol{W}_{q}\right|^{2}-2\left|m e^{j \alpha} \boldsymbol{W}_{q}\right|\left|\boldsymbol{W}_{p}\right| \cos \delta
\end{aligned}
$$

and

$$
\begin{aligned}
\frac{D_{(2)}^{2}}{\left|s_{i}\right|^{2}} & =\left|\boldsymbol{W}_{p}-e^{j \alpha} \boldsymbol{W}_{q}\right|^{2}=\left|\boldsymbol{W}_{p}\right|^{2} \\
& +\left|e^{j \alpha} \boldsymbol{W}_{q}\right|^{2}-2\left|e^{j \alpha} \boldsymbol{W}_{q}\right|\left|\boldsymbol{W}_{p}\right| \cos \delta .
\end{aligned}
$$

Using $\left|W_{p}\right|=\left|W_{q}\right|=1$ and subtracting (18) from (17) follows

$$
\begin{aligned}
\frac{D_{(3)}^{2}-D_{(2)}^{2}}{\left|s_{i}\right|^{2}} & =m^{2}-2 m \cos \delta-1+2 \cos \delta \\
& =(m-1)(m+1-2 \cos \delta)
\end{aligned}
$$

As $m>1$, it implies $D_{(3)}^{2}>D_{(2)}^{2}$. Hence, the minimum distance in case (3) is always greater than the minimum distance in case (2) and, therefore, it is not relevant for the maximization of the minimum distance.

After studying all three cases, it can be concluded that the maximization of the minimum distance between possible GLPSM symbols is achieved for the phases given in (13).

The proposed scheme is regarded as SS-GLPSM and is a straightforward generalization of LPSM proposed in [16] and LPSM can be considered as a special case SSGLPSM for the cases of two and four transmit antennas.

As mentioned earlier, MMSE receiver is used in this paper. Therefore, after MMSE equalization is performed [16], the receiver creates the vector of $N_{T}$ equalized signals and finds the nearest possible GLPSM symbol. The number of possible GLPSM symbols is $2^{L+K}$, hence the receiver calculates $2^{L+K}$ possible distances to make a decision. This decisioning is performed per subcarrier per SC-FDMA symbol.

As an example, the case of eight transmit antennas is presented in Appendix B. From (1) and (12), $N_{T}=8 \mathrm{im}$ plies that $a_{t, p}=\sqrt{\frac{1}{8}} e^{j \phi_{t, p}}$ where $\phi_{t, p}=\frac{2 \pi(t-1) p}{8}$ for $t=1,2$, $\ldots, 8$ and $p=0,1, \ldots, 7$. The coding coefficients, $a_{t, p}$, for this case are presented in Table 3 in Appendix B.

However, as concluded previously, the rotation of all phases of one spatial symbol by any phase shift does not affect its distances from other spatial symbols hence any spatial symbol can be further rotated. For the sake of clarity, we do not observe any phase shifts in this case and all spatial symbols have the same phase on the first antenna $(t=1)$.

Furthermore, regarding the Table 3 from Appendix B, if, e.g., QAM is used and $s_{[00]}=e^{j \frac{\pi}{4}}$ (corresponding to the transmission of bits [00]) and $p=5$ (corresponding to the transmission of bits [101] in spatial components), the transmitter sends five bits by sending the signal:

$$
\boldsymbol{v}_{5,[00]}=s_{[00]} W_{5}=\sqrt{\frac{1}{8}}\left[e^{j \frac{\pi}{4}}, e^{j \frac{3 \pi}{2}}, e^{j \frac{3 \pi}{4}}, 1, e^{j \frac{5 \pi}{4}}, e^{j \frac{\pi}{2}}, e^{j \frac{7 \pi}{4}},-1\right]
$$

via its eight transmit antennas.

As MMSE is used, the receiver creates the vector of eight equalized signals and finds the nearest possible GLPSM symbol. The number of possible GLPSM symbols is $2^{5}=32$, hence the receiver calculates 32 possible distances to make a decision about transmitted QAM symbol and spatial bits.

\section{Generalized single-stream GLPSM}

As shown in Section 2, SS-GLPSM transmitter with $N_{T}=2^{K}$ transmit antennas conveys $K$ bits in the spatial component. Hence, the number of transmit antennas must be doubled in order to add another bit in the spatial component. To overcome this limitation, we propose GSS-GLPSM technique that places more than $K$ bits in the spatial component. The system model is the same as in Fig. 1.

Unlike $p=0,1, \ldots, N_{T}-1$, defined in Section 2, we define $p_{G}=0,1, \ldots, 2^{P}-1$, where $P$ is the number of bits to be placed in the spatial component, i.e., the spatial bits. In this case, we consider $P>K$, hence the number of spatial symbols is increased from $N_{T}$ to $2^{P}$ and the number of spatial bits per spatial symbol is increased from $K$ to $P$. Using the approach from Section 2, in analogy to (1), we define

$$
a_{t, p_{G}}=\sqrt{\frac{1}{N_{T}}} e^{j \bar{\phi}_{t, p_{G}}}
$$

where, in analogy to (13), 


$$
\bar{\phi}_{t, p_{G}}=\frac{2 \pi(t-1) p_{G}}{2^{P}} .
$$

The previous section has proven the optimality of these definitions in the case $P=K$, while the empirical results, provided in Section 6, will justify the analogous definitions for $P>K$. Using this approach, $P$, instead of $K$, spatial bits are transmitted via spatial symbol. Hence, the number of spatial symbols is increased for the same number of transmit antennas. In comparison to SSGLPSM for the same overall bitrate, as more bits are transmitted via spatial component less bits are transmitted via QAM/PSK symbols and more robust QAM/PSK modulation can be used.

The difference, in comparison to SS-GLSPM, is in the number of spatial symbols and the constellation size, hence the receiver for GSS-GLPSM is the same as in SSGLPSM. Only simple linear MMSE receivers [16] are considered in this paper.

\section{Multiple stream GLPSM}

Another approach for avoiding the limitation observed in Section 2 is to combine GLPSM with spatial multiplexing [21]. In this case, multiple streams are generated and SS-GLPSM is used for each of them. This approach is proposed in this section as MS-GLPSM. The block scheme of the MS-GLPSM transmitter is given in Fig. 2.

In this technique, the set of $N_{T}$ transmit antennas is divided into $S$ subsets of $N_{S}$ antennas, i.e., $N_{T}=S N_{S}$. Further, each of the subsets is used for independent SSGLPSM coding, discussed in Section 2, and carries independent QAM/PSK symbol sequence. If $N_{s}=2^{K_{s}}$, then each subset carries $K_{S}$ bits in the spatial component. For simplicity, it can be assumed that all QAM/PSK sequences use the same modulation that maps $L$ bits to the QAM/PSK symbol. The number of subsets, $S$, presents the number of streams. The input bit sequence, consisting of $M S\left(L+K_{S}\right)$ bits, is divided into $S$ streams of $M\left(L+K_{S}\right)$ bits that are further coded independently according to SS-GLPSM described in Section 2. Hence, MS-GLPSM conveys $S\left(L+K_{S}\right)$ bits per subcarrier, whereas SS-GLPSM carries $L+K$.

It is worth noting that, as GLPSM is regarded as an open-loop MIMO technique, the transmitter has no knowledge of the conditions in the channel, hence it is irrelevant how the grouping of antennas into the subsets is performed. For the simplicity, it is assumed that antennas $t=1,2, \ldots, N_{S}$ belong to the first subset, antennas $t=N_{S}+1, N_{S}+2, \ldots, 2 N_{S}$ belong to the second subset, etc. If $N_{R}$ denotes the number of receive antennas, then all $S$ streams are received on all $N_{R}$ antennas.

Unlike SS-GLPSM and GSS-GLPSM, this technique suffers from the interference between the streams, as it is the case in spatial-multiplexing techniques, such as $\mathrm{V}$ BLAST (vertical-Bell Laboratories layered space-time) [21, 22]. Therefore, besides using a simple MMSE receiver, a significant performance gain can be achieved using the receivers with interference cancelation techniques, well-known from V-BLAST [21-24], that can be applied to MS-GLSPM receiver in a similar fashion. In this paper, we studied ordered successive interference cancelation (OSIC) technique and, in accordance to MMSE receivers considered in Sections 3 and 4, MMSE equalization with OSIC is considered.

In accordance to V-BLAST [21, 22], the OSIC receiver performs a simple linear MMSE equalization to obtain

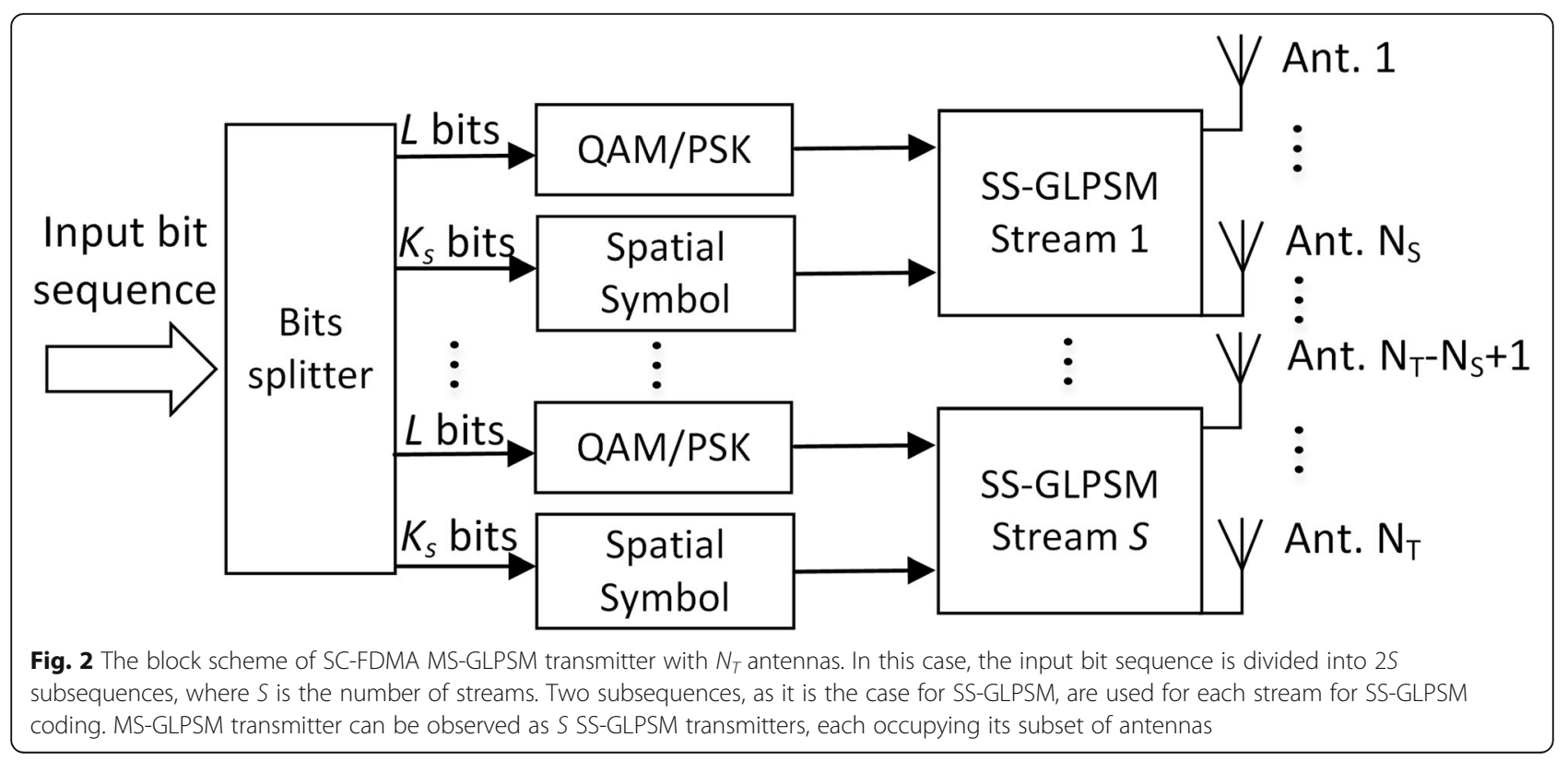


the initial decision. For OSIC receiver, it is necessary to have an ordering criterion, hence the streams are ordered in descending order starting from the strongest stream. Let $\boldsymbol{H}^{(i)}$ denote an $N_{R} \times N_{T}$ subchannel matrix for the $i$ th subcarrier and its elements, $H_{r, t}^{(i)}$, denote the complex channel gain on the $i$ th subchannel between $t$ th transmit and $r$ th receive antenna. Further, let $\boldsymbol{G}^{(i)}$ denote the corresponding equalization matrix of matrix $\boldsymbol{H}^{(i)}$. As MMSE is considered in this paper, we use the approach similar to [22]:

$$
\boldsymbol{G}^{(i)}=\left(\boldsymbol{H}^{(i)^{\mathrm{H}}} \boldsymbol{H}^{(i)}+\frac{1}{S N R} \mathcal{I}_{N_{T}}\right)^{-1} \boldsymbol{H}^{(i)^{\mathrm{H}}}
$$

where $S N R$ is the signal-to-noise ratio, assumed to be known at the receiver side. The matrix $G^{(i)}$ is of size $N_{T} \times N_{R}$ and let $G_{t, r}^{(i)}$ denote the elements of the matrix $\boldsymbol{G}^{(i)}$. The elements $G_{t, r}^{(i)}$ are the equalization coefficients for $i$ th subcarrier on the channel between $t$ th transmit and $r$ th receive antenna. Let $\boldsymbol{G}_{s}^{(i)}$ denote the submatrix of $G^{(i)}$ of size $N_{S} \times N_{R}$ for sth stream, where $s=1, \ldots, S$. As sth stream spans over antennas $t=(s-1) N_{S}+1, \quad(s-1) N_{S}+2, \ldots, s N_{S}$, the submatrix $\boldsymbol{G}_{s}^{(i)}$ consists of these rows. The stream is considered as the strongest stream if the sum of its squared Frobenius norms over all subcarriers is the lowest. For the sth stream:

$$
\Theta_{s}=\sum_{i=1}^{M}\left\|G_{s}^{(i)}\right\|_{\mathrm{F}}^{2}=\sum_{i=1}^{M}\left(\sum_{t=(s-1) N_{S}+1}^{s N_{S}} \sum_{r=1}^{N_{R}}\left|G_{t, r}^{(i)}\right|^{2}\right)
$$

denotes the ordering coefficient that corresponds to the sum of squared absolute values of all subchannel equalization coefficients of the observed stream. For the stream with greater absolute channel gains, the equalization coefficients are lower. The part of (24) inside the brackets presents the squared Frobenius norm of the submatrix $\boldsymbol{G}_{s}^{(i)}$. Unlike OFDM, each subcarrier in SC-FDMA carries all QAM/PSK symbols, hence it is necessary to sum over all subcarriers.

After obtaining the ordering coefficients, the strongest stream is detected first and its decision with corresponding channels is subtracted from the received signals. This process is repeated until all streams are detected [21-23]. The obtained coefficients cover the duration of one SC-FDMA symbol, consisting of $M S\left(L+K_{S}\right)$ bits. Therefore, the calculation of the coefficients in (24) and the ordering is performed per SC-FDMA symbol.

\section{Discussion and results}

\subsection{PAPR comparison}

In order to evaluate the proposed techniques, in this section we compared the PAPR curves of the proposed techniques with single transmit antenna SCFDMA and OFDM, as their PAPR curves are well known [4].

In this paper, discrete-time $P A P R[19,25]$ is considered. If the discrete-time sampled transmitted signal is presented as a vector $\boldsymbol{x}=\left[x_{1}, x_{2}, \ldots, x_{N}\right]^{T}$, then PAPR of the signal can be defined as

$$
\operatorname{PAPR}(\boldsymbol{x})=\frac{\max _{1 \leq n \leq N}\left|x_{n}\right|^{2}}{\mathrm{E}\left\{\left|x_{n}\right|^{2}\right\}} .
$$

The PAPR is usually measured using the complementary cumulative distribution function (CCDF) [25]. CCDF expresses the probability that $P A P R$ is greater than the level $P A P R_{0}$ :

$$
\operatorname{CCDF}\left(P A P R_{0}\right)=\operatorname{Pr}\left(P A P R>P A P R_{0}\right) .
$$

For the comparison, 16-QAM underlying modulation was used and the user occupied $M=96$ subcarriers (or eight LTE resource blocks) out of $N=512$ (5 MHz LTE channel). No shaping filters were used.

Figure 3 presents PAPR curves for the proposed SSGLPSM, GSS-GLPSM, and MS-GLPSM scheme for $N_{T}=8$ transmit antennas in comparison to OFDM and SCFDMA.

Although all antennas for the proposed techniques have similar PAPR curves, for the sake of clarity, only antenna 3 is presented. It can be concluded that all three techniques maintained the PAPR levels of the underlying QAM/PSK modulation, i.e., 16-QAM. As SC-FDMA signal is the oversampled QAM/PSK signal, the conclusion is that the PAPR level for any of the three proposed GLPSM techniques is determined by the underlying modulation and it is not affected by the GLPSM technique. Furthermore, the number of streams in MS-GLPSM does not affect the PAPR levels.

However, it is worth noting that the overall data rates in the observed cases are different. SS-GLSPM conveys $K=3$ bits in the spatial component, because $N_{T}=8$, hence its overall data rate is 7 bits per subcarrier per SC-FDMA symbol. GSS-GLPSM places $P=4$ bits in the spatial component achieving 8 bits per subcarrier per SC-FDMA symbol overall data rate. For MS-GLPSM, the cases with $S=2$ and $S=4$ are considered. For $S=2$, both streams convey $K_{S}=2$ bits in the spatial component, resulting in $2(2+4)=12$ bits per subcarrier per SC-FDMA symbol. For $S=4$, $K_{S}=1$ bit is placed in the spatial component, resulting 


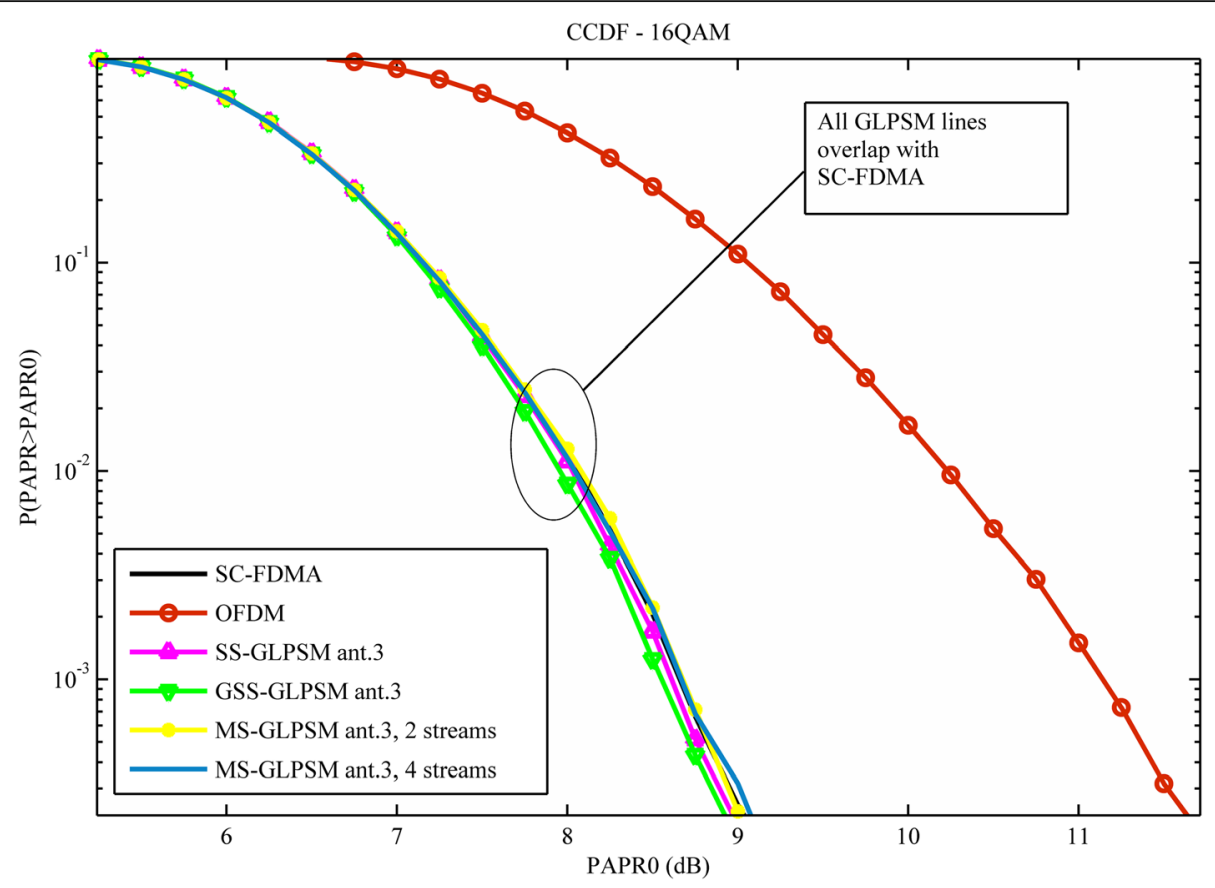

Fig. 3 PAPR comparison for SC-FDMA GLPSM in the case of eight transmit antennas. The figure presents the PAPR comparison for all three proposed GLPSM techniques (SS-GSLPM, GSS-GLPSM, and MS-GLPSM) and the conventional SC-FDMA and OFDM, given as the references. All transmit antennas have similar PAPR curves, hence, for the sake of clarity, only curves for antenna 3 are presented. In all observed techniques, the underlying modulation is 16-QAM. It can be concluded that all three proposed techniques maintain the same PAPR level as the conventional SCFDMA and that their PAPR levels are determined by the underlying modulation. Furthermore, MS-GLPSM maintains the same PAPR level regardless of the number of streams used.

in $4(1+4)=20$ bits per subcarrier per SC-FDMA symbol. This implies that PAPR curves are determined by the underlying QAM/PSK modulation and not by the GLPSM technique or the number of streams.

\subsection{Performance comparison}

After confirming that the PAPR levels are unchanged when GLPSM is applied to SC-FDMA, the performance comparison is performed. In order to have a fair comparison, data rates in this case are the same for all techniques. Furthermore, the equal number of transmit and receive antennas in all techniques is considered. For the sake of simplicity, it is assumed that the system has perfect synchronization and the receiver has perfect channel estimation.

The conventional V-BLAST [21], implemented in SCFDMA, is observed as a well-known spatial multiplexing technique. The implementation of spatial multiplexing with successive interference cancelation receivers in SC-FDMA is covered in [26-28]. The transmitter splits QAM/PSK sequence into $N_{T}$ subsequences and performs $N_{T}$ separate SC-FDMA modulations. Each subsequence is transmitted from its antenna. All subsequences are placed on the same block of subcarriers and transmitted simultaneously from the separate antennas, hence pose the interference to each other. For clarity, we used an approach similar to the receiver presented in Section 4. Therefore, at the receiver side, after simple linear equalization, e.g., MMSE, the strongest component is detected and subtracted from the received signals. This process is repeated until all $N_{T}$ subsequences are detected. Similarly to (24), the strongest transmit antenna is obtained after calculating the ordering coefficients:

$$
\Theta_{t}=\sum_{i=1}^{M}\left\|G_{t}^{(i)}\right\|_{F}^{2}=\sum_{i=1}^{M}\left(\sum_{r=1}^{N_{R}}\left|G_{t, r}^{(i)}\right|^{2}\right)
$$

where $t=1,2, \ldots, N_{T}$ is the index of the subsequence, or corresponding transmit antenna, and $\boldsymbol{G}_{t}^{(i)}$ denotes $t$ th row of the matrix $\boldsymbol{G}^{(i)}$ given in (23). The transmit antenna with the lowest $\Theta_{t}$ is selected as the strongest. Therefore, V-BLAST model can be seen as an MSGLPSM model with $N_{T}$ streams and each stream is conveyed via its single assigned antenna, hence without the bits transmitted in the spatial domain. 
The first comparison is performed in EVA-70 (Extended Vehicular A) [29] channel model for the case of $8 \times 12$ MIMO. As SC-FDMA is used in LTE uplink, it is expected that the receiver, a base station, will accommodate a greater number of antennas than the transmitter, hence $N_{R}=12$ is selected. In this, all other comparisons, it is assumed that the channels are uncorrelated. The overall data rates are equal in all cases to 8 bits per subcarrier per SC-FDMA symbol and the parameters of the observed models are given in Table 1.

As given in previous sections, the parameters $K$ and $P$ present the number of spatial bits, whereas $K_{S}$ is the number of spatial bits per stream.

In all six models, the simple linear MMSE receiver is considered. The user occupied $M=60$ subsequent subcarriers (or 5 LTE resource block) out of $N=512$. The performance comparison is obtained via simulations and presented in Fig. 4. The numbers in brackets, given in Figs. 4, 5, 6, and 7, are the numbers of models given in the corresponding tables.

It can be concluded that the models 1,2 , and 3 with single stream GLPSM show significantly poorer performances than the other models. The model 2 with 4 bits in the spatial domain and 4 bits in the constellation achieves the best performances of all single stream GLSPM models, around $1 \mathrm{~dB}$ better than the other techniques. A significant performance gain is achieved using multiple streams and the model 4 with two streams shows performances around $1 \mathrm{~dB}$ better than V-BLAST. For the models 4 and 5, substreams have two and four antennas per stream, respectively, hence LPSM [16] coding, the special case of SS-GLPSM, is used.

It is worth noting that the single stream GLPSM techniques require higher order underlying modulations that, in general, have greater PAPR values [30, 31]. Hence, for better evaluation of the techniques, the PAPR comparison of the models is given in Fig. 5 and, for the sake of clarity, only antenna 3 is presented. It can be noted that PAPR curves are determined by the underlying modulation, hence PAPR curves for models 1 and 2 overlap and the curves for

Table 1 Models parameters

\begin{tabular}{lllll}
\hline$N_{T}=8, N_{R}=12,8$ bits/subcarrier/symbol & & \\
\hline Model & Technique & $L$ & $K_{S}$ & 5 \\
\hline 1 & SS-GLPSM & 5 (32-QAM) & 3 & 1 \\
2 & GSS-GLPSM & 4 (16-QAM) & 4 & 1 \\
3 & GSS-GLPSM & 3 (8-PSK) & 5 & 1 \\
4 & MS-GLPSM & 2 (QPSK) & 2 & 2 \\
5 & MS-GLPSM & 1 (BPSK) & 1 & 4 \\
6 & V-BLAST & 1 (BPSK) & 0 & 8 \\
\hline
\end{tabular}

the model 3 and 4 , and 5 and 6 respectively. The main conclusion is that MS-GLPSM shows better performance than the other observed techniques (Fig. 4), including V-BLAST, and maintains good PAPR curves (Fig. 5).

LTE specifications define QPSK (quadrature PSK) and 16-QAM, hence we introduced 32-QAM, 8-PSK, and BPSK (binary PSK) in a similar fashion. In order to avoid transitions through zero in BPSK, that causes PAPR increase, in this paper we considered $\pi / 2$ BPSK, as it was defined in the earlier LTE specifications [32]. Hence, the models 5 and 6 utilize $\pi / 2$ BPSK that achieves the same performances as the conventional BPSK for significantly lower PAPR value.

As mentioned in Section 5, when multiple streams exist, a significant performance gain can be achieved using OSIC receiver. In the performance comparison presented in Fig. 6, simple MMSE and OSIC receivers for the models 4, 5, and 6 from Table 1 are considered. In this case, ETU-70 (extended typical urban) [29] channel model is considered, with all the other simulation parameters same as in the previous comparison. Singlestream GLPSM techniques are not considered as they cannot benefit from OSIC receiver.

The model 4, MS-GLPSM with two streams, shows better performance than V-BLAST for both types of receivers, whereas MS-GLPSM with four streams is slightly worse. A significant performance gain is achieved when OSIC receiver is used, for all three models.

In the last comparison, lower data rate of 6 bits per subcarrier per SC-FDMA symbol is considered. In this case, V-BLAST is not considered, only the proposed techniques are compared, and the results are presented in Fig. 7. The simulation parameters are shown in Table 2. EPA-5 (extended pedestrian A) [29] channel model is used and the channels are uncorrelated.

In this case, SS-GLPSM with simple MMSE achieves performances similar to MS-GLSPM with OSIC receiver and is significantly better, around $1 \mathrm{~dB}$, than the two other techniques with the same type of receiver. However, it is worth noting that the PAPR curves are determined by the underlying QAM/PSK technique, therefore it implies that model 3 has better PAPR curves that the first two models.

\section{Conclusion}

This paper presents GLPSM, a low PAPR SC-FDMA MIMO technique. After presenting three implementations, SS-GLSPM, GSS-GLPSM, and MS-GLPSM, it is confirmed via simulations that the low PAPR level is maintained on all transmit antennas for all techniques. In general, the best technique of the three proposed depends on the overall data rate. For higher 


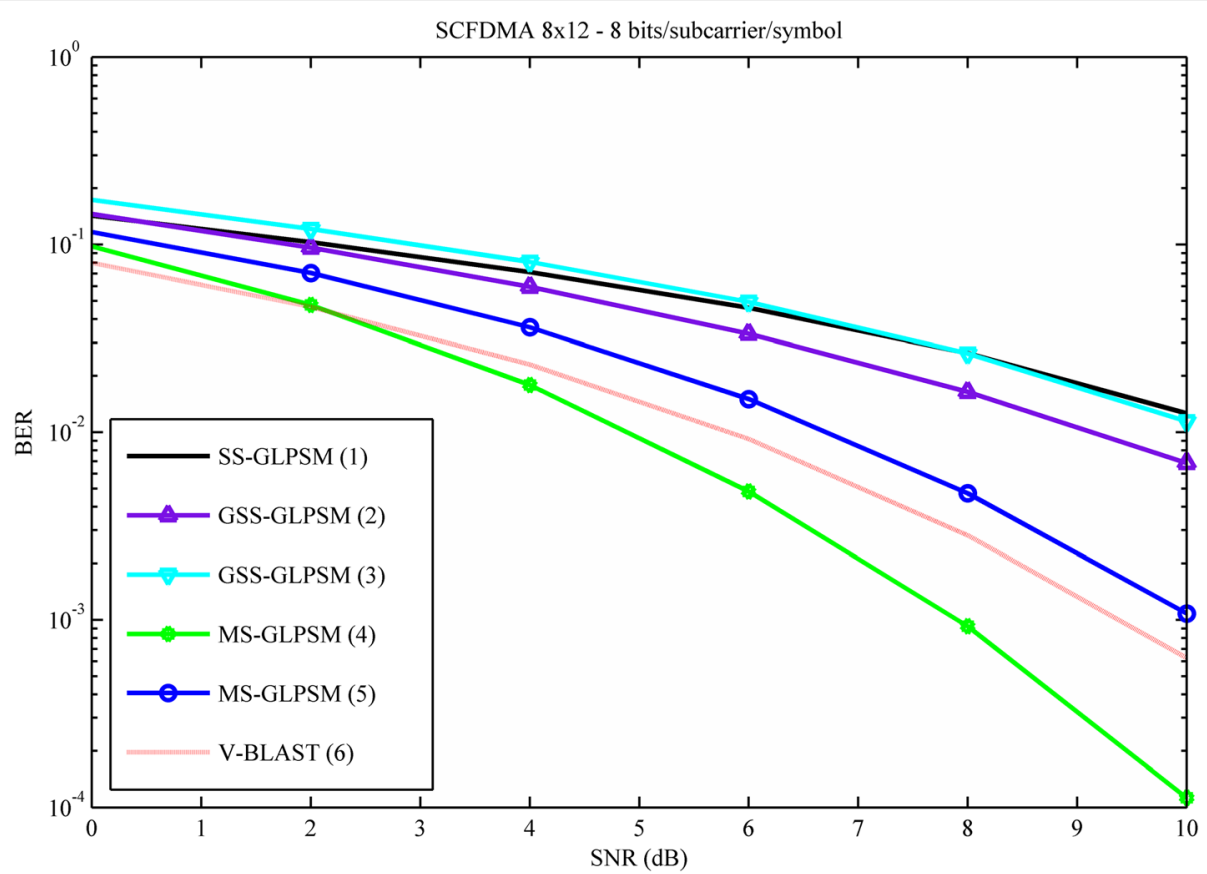

Fig. 4 Performance comparison for SC-FDMA GLPSM and SC-FDMA V-BLAST with the parameters given in Table 1. The performance comparison of the three proposed techniques and SC-FDMA with V-BLAST, given as a reference. The comparison is given with the constraint of the same overall data rate of 8 bits/subcarrier/symbol. $8 \times 12$ MIMO in EVA-70 is observed. MMSE receiver is observed in all cases

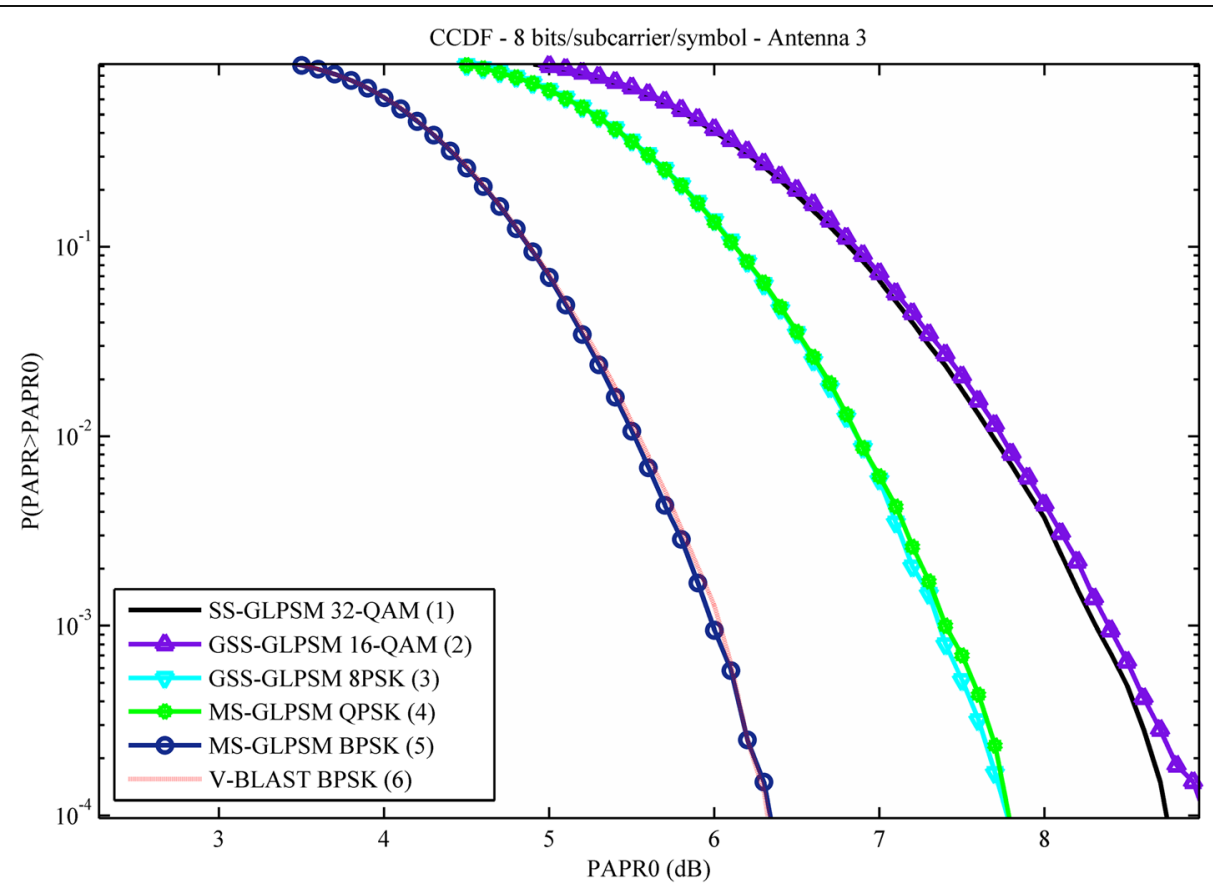

Fig. 5 PAPR comparison for SC-FDMA GLPSM and SC-FDMA V-BLAST with the parameters given in Table 1. In order to have a full comparison of the techniques given in Table 1, after performance comparison presented in Fig. 4. PAPR comparison of the techniques is given. As the PAPR curves are determined by the underlying modulation, SS-GLPSM and GSS-GLPSM have higher PAPR levels because high order modulations have to be used. Techniques with multiple streams, MS-GLPSM or V-BLAST, utilize low order modulations that inherently provide better PAPR curves 


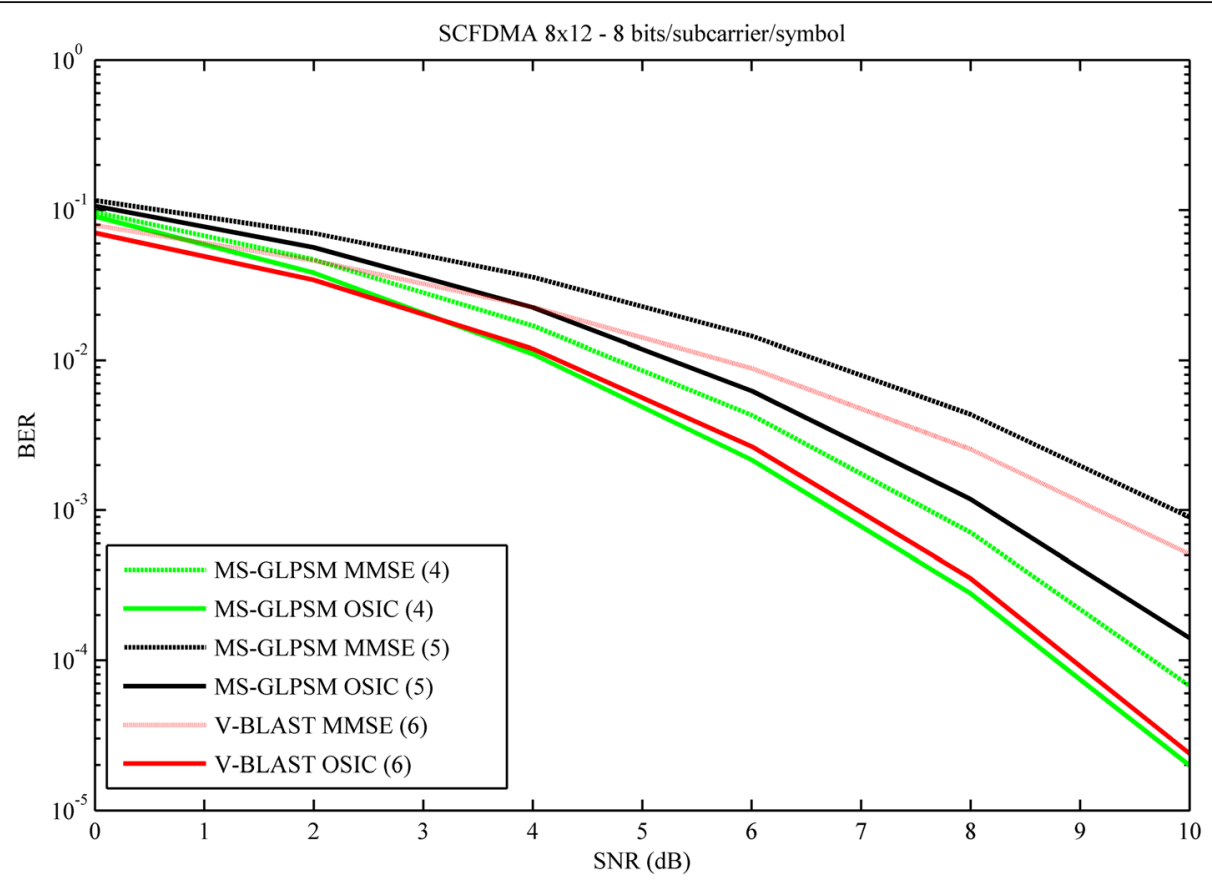

Fig. 6 Performance comparison for MS-GLPSM and V-BLAST for MMSE and OSIC receivers. As the significant performance gain in the cases of multiple streams can be achieved using OSIC receiver, the performance comparison is given for MS-GLPSM SC-FDMA and V-BLAST SC-FDMA when MMSE or OSIC receivers are used. The cases of two and four streams are presented for MS-GLPSM. All parameters are given in Table 1. Channel model is ETU-70

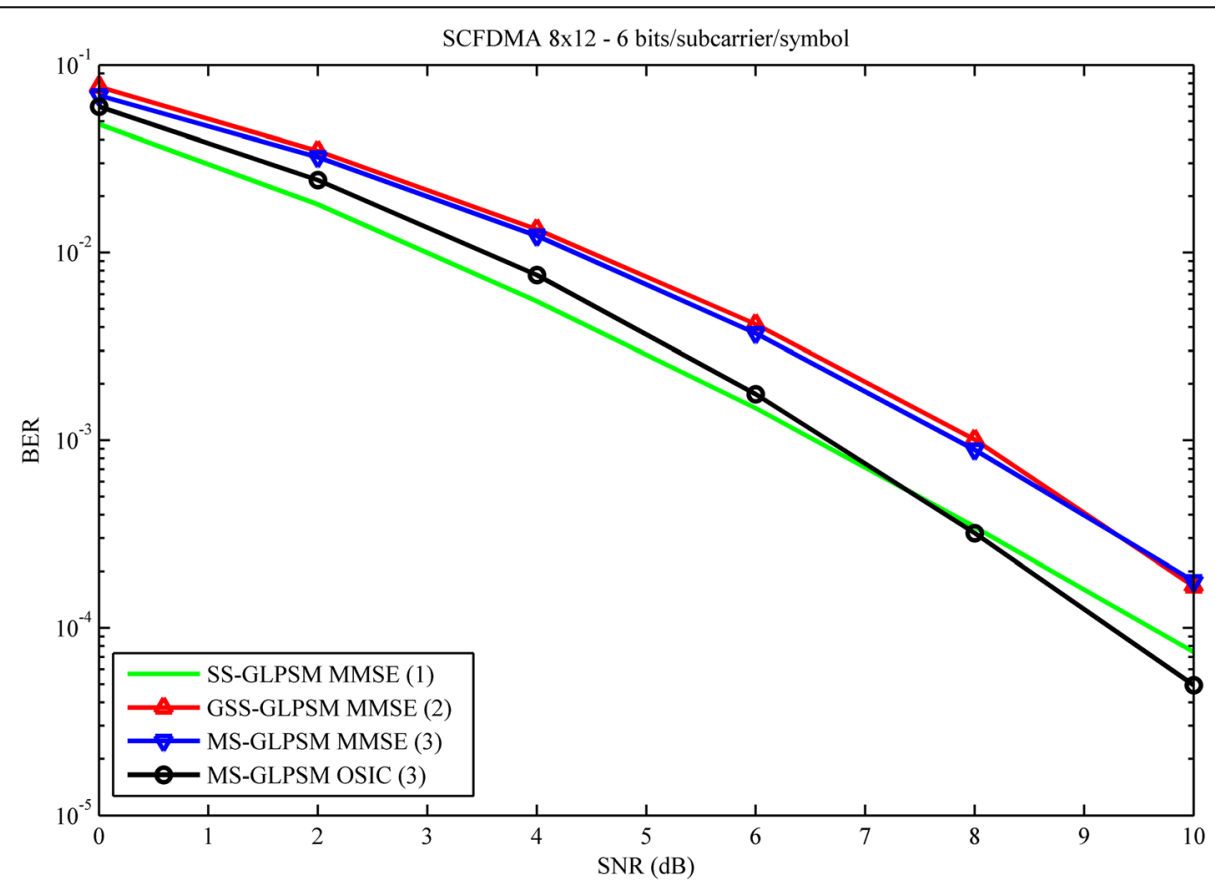

Fig. 7 Performance comparison for SC-FDMA GLPSM techniques with the parameters given in Table 2. This performance comparison is given for lower over datarate of 6 bits/subcarrier/symbol. Because of that, V-BLAST is omitted and only the proposed techniques are compared. For MSGLPSM, MMSE and OSIC receiver are compared. $8 \times 12$ MIMO in EPA-5 is observed 
Table 2 Models parameters

\begin{tabular}{lllll}
\hline$N_{T}=8, N_{R}=12,6$ bits/subcarrier/symbol & & \\
\hline Model & Technique & $L$ & $K, P, K_{S}$ & $S$ \\
\hline 1 & SS-GLPSM & 3 (8-PSK) & 3 & 1 \\
2 & GSS-GLPSM & 2 (QPSK) & 4 & 1 \\
3 & MS-GLPSM & 1 (BPSK) & 2 & 2 \\
\hline
\end{tabular}

data rates, single stream techniques require large, hence less robust, underlying modulations and show significantly poorer performance than V-BLAST or MS-GLPSM. Furthermore, high order underlying modulations have higher PAPR values. The best performances are achieved when the same number of bits is transmitted in the spatial component and in the constellation symbol. Therefore, single stream techniques show good performances in low data rate cases and PAPR values are acceptable.

MS-GLPSM splits antennas into subsets and achieves better performances in the cases of higher data rates. It even shows better performance than $\mathrm{V}$ BLAST. As it is the case in V-BLAST, a significant performance gain can be achieved using more complex OSIC receiver.

\section{Appendix A}

Theorem: $N_{T}$ vectors, given in (12), with the phases given in (13) are vertices of a regular simplex with the centroid in the origin.

Proof:

Firstly, using the fact that all vertices are points on the sphere centered in the origin, it can be shown that the centroid of the polytope is in the origin, since the arithmetic mean for any coordinate is

$$
\begin{aligned}
C_{t}^{o d d} & =\frac{\sqrt{\frac{1}{N_{T}} \sum_{p=1}^{N_{T}} \cos \frac{2 \pi(t-1) p}{N_{T}}}}{N_{T}}=0 \\
C_{t}^{\text {even }} & =\frac{\sqrt{\frac{1}{N_{T}} \sum_{p=1}^{N_{T}} \sin \frac{2 \pi(t-1) p}{N_{T}}}}{N_{T}}=0
\end{aligned}
$$

where $C_{t}^{o d d}$ is a coordinate on the odd position of the centroid and $C_{t}^{e v e n}$ is on the even position.

As the centroid is located in the origin, it can be easily shown that the distances of all vertices from the centroid are equal and they are equal to the radius of the sphere, i.e., for every $p$,

$$
\left|\boldsymbol{W}_{p}^{\left[2\left(N_{T}-1\right)\right]}\right|=\sqrt{\frac{N_{T}-1}{N_{T}}} .
$$

It is worth noting that, although the space is $2\left(N_{T}-1\right)$ dimensional, the simplex is $N_{T}-1$ dimensional as it has $N_{T}$ vertices. This implies that a regular simplex is formed when the angle subtended by any two vertices through the centroid is equal to $\cos ^{-1} \frac{-1}{N_{T}-1}$.

In order to prove this, the scalar product of any two vectors $\boldsymbol{W}_{p}^{\left[2\left(N_{T}-1\right)\right]}$ and $\boldsymbol{W}_{q}^{\left[2\left(N_{T}-1\right)\right]}$, where $p, q=0,1, \ldots$, $N_{T}-1$ and $p \neq q$, is observed:

$$
\begin{aligned}
W_{p}^{\left[2\left(N_{T}-1\right)\right]} \cdot W_{q}^{\left[2\left(N_{T}-1\right)\right]} & =\frac{1}{N_{T}} \sum_{t=2}^{N_{T}}\left(\cos \frac{2 \pi(t-1) p}{N_{T}} \cos \frac{2 \pi(t-1) q}{N_{T}}\right. \\
& \left.+\sin \frac{2 \pi(t-1) p}{N_{T}} \sin \frac{2 \pi(t-1) q}{N_{T}}\right) \\
& =\frac{1}{N_{T}} \sum_{t=2}^{N_{T}} \cos \frac{2 \pi(t-1)(p-q)}{N_{T}} .
\end{aligned}
$$

It follows that

$$
W_{p}^{\left[2\left(N_{T}-1\right)\right]} \cdot W_{q}^{\left[2\left(N_{T}-1\right)\right]}=\frac{1}{N_{T}}\left(\sum_{t=1}^{N_{T}} \cos \frac{2 \pi(t-1)(p-q)}{N_{T}}-1\right) .
$$

The proposed phases (13) satisfy the condition (9), because the differences of phases present the angles of the vertices of the regular polygon centered in the origin. It implies that the first part in brackets in (32) equals 0 , hence it follows that

$$
\boldsymbol{W}_{p}^{\left[2\left(N_{T}-1\right)\right]} \cdot \boldsymbol{W}_{q}^{\left[2\left(N_{T}-1\right)\right]}=-\frac{1}{N_{T}} .
$$

Using (30), the angle between the two vectors, $\Phi_{p}$, q, can be obtained from

$$
\begin{aligned}
\cos \varangle \Phi_{p, q} & =\frac{\boldsymbol{W}_{p}^{\left[2\left(N_{T}-1\right)\right]} \cdot \boldsymbol{W}_{q}^{\left[2\left(N_{T}-1\right)\right]}}{\left|\boldsymbol{W}_{p}^{\left[2\left(N_{T}-1\right)\right]}\right|\left|\boldsymbol{W}_{q}^{\left[2\left(N_{T}-1\right)\right]}\right|} \\
& =-\frac{1}{N_{T}-1} .
\end{aligned}
$$

From (28), (29), (30), and (34), it can be concluded that $N_{T}$ points form a regular simplex for the phases proposed in (13).

Therefore, the maximization of the minimum distance is achieved for the phases given in (13).

\section{Appendix B}

The coding coefficients, $a_{t, p}$, for SS-GLSPM for the case of of eight transmit antennas is presented in Table 3. 
Table 3 GLPSM coding coefficients for $N_{T}=8$

\begin{tabular}{|c|c|c|c|c|c|c|c|c|}
\hline & $t=1$ & $t=2$ & $t=3$ & $t=4$ & $t=5$ & $t=6$ & $t=7$ & $t=8$ \\
\hline$p=0$ & $\sqrt{\frac{1}{8}}$ & $\sqrt{\frac{1}{8}}$ & $\sqrt{\frac{1}{8}}$ & $\sqrt{\frac{1}{8}}$ & & & $\sqrt{\frac{1}{8}}$ & \\
\hline$p=1$ & & & & & & & & \\
\hline$p=2$ & & & & & & & & \\
\hline$p=3$ & & & & & & & & \\
\hline$p=4$ & $\sqrt{1}$ & & & & & & & \\
\hline$p=5$ & & & & & & & & \\
\hline$p=6$ & & & & & & & & \\
\hline$p=7$ & $\sqrt{\frac{1}{8}}$ & $\sqrt{\frac{1}{8}} e$ & $\sqrt{\frac{1}{8}} e$ & & $-\sqrt{\frac{1}{\varepsilon}}$ & & $\sqrt{\frac{1}{8}}$ & \\
\hline
\end{tabular}

\section{Abbreviations}

BPSK: Binary phase shift keying; CCDF: Complementary cumulative distribution function; EPA: Extended pedestrian A; ETU: Extended typical urban; EVA: Extended vehicular A; FFT: Fast Fourier transform; GLPSM: Generalized low peak-to-average power ratio spatial modulation; GSM: Generalized spatial modulation; GSS-GLPSM: Generalized single-stream GLPSM; IFFT: Inverse fast Fourier transform; LPSM: Low peak-to-average power ratio spatial modulation; LTE: Long-term evolution; MIMO: Multipleinput multiple-output; MMSE: Minimum mean-square error; MSGLPSM: Multiple-stream GLPSM; OFDM: Orthogonal frequency division multiplexing; OSIC: Ordered successive interference cancelation; PAPR: Peakto-average power ratio; PSK: Phase shift keying; QAM: Quadrature amplitude modulation; QPSK: Quadrature phase shift keying; RF: Radiofrequency; SCFDMA: Single-carrier frequency division multiple access; SM: Spatial modulation; SS-GLPSM: Single-stream GLPSM; V-BLAST: Vertical-Bell Laboratories layered space-time

\section{Acknowledgments}

The authors acknowledge the support of their employers.

\section{Authors' contributions}

DS and GS proposed and designed the techniques. ASK and ZI proved the optimal coefficient selection. DS and GS performed the simulations. All authors read and approved the final manuscript.

\section{Authors' information}

Not applicable.

\section{Funding}

No funding

\section{Availability of data and materials}

The data used to support the findings of this study are available from the corresponding author upon request.

\section{Competing interests}

The authors declare that they have no competing interests.

\section{Author details}

${ }^{1} \mathrm{NOSBIH}$, Sarajevo, Bosnia and Herzegovina. ${ }^{2}$ Faculty of Electrical Engineering and Computing, University of Zagreb, Zagreb, Croatia.

\section{Received: 7 February 2019 Accepted: 13 February 2020}

Published online: 19 February 2020

\section{References}

1. R. Mesleh, H. Haas, C.W. Ahn, S. Yun, in Proc. of First International Conference on Communications and Networking in China, Spatial modulation - a new low complexity spectral efficiency enhancing technique (2006), pp. 1-5. https://doi.org/10.1109/CHINACOM.2006.344658

2. R. Mesleh, H. Haas, S. Sinanović, C.W. Ahn, S. Yun, Spatial modulation. IEEE Trans. Veh. Technol. 57(4), 2228-2241 (2008). https://doi.org/10.1109/TVT. 2007.912136

3. S. Sugiura, L. Hanzo, Single-RF Spatial modulation requires single-carrier transmission: frequency-domain turbo equalization for dispersive channels. IEEE Trans. Veh. Technol. 64(10), 4870-4875 (2015). https://doi.org/10.1109/ TVT.2014.2370679

4. A. Younis, N. Serafimovski, R. Mesleh, H. Haas, in Generalised spatial modulation. in Proc. of Conference Record of the Forty Fourth Asilomar Conference on Signals, Systems and Computers (2010), pp. 1498-1502. https://doi.org/10.1109/ACSSC.2010.5757786

5. J. Fu, C. Hou, W. Xiang, L. Yan, Y. Hou, in GLOBECOM Workshops, Generalised spatial modulation with multiple active transmit antennas (2010), pp. 839844. https://doi.org/10.1109/GLOCOMW.2010.5700442

6. J. Wang, S. Jia, J. Song, Generalised spatial modulation system with multiple active transmit antennas and low complexity detection scheme. IEEE Trans. Wireless Commun. 11(4), 1605-1615 (2012). https://doi.org/10.1109/TWC. 2012.030512 .111635

7. T. Lakshmi Narasimhan, P. Raviteja, A. Chockalingam, Generalized spatial modulation in large-scale multiuser MIMO Systems. IEEE Trans. Wireless Commun. 14(7), 3764-3779 (2015). https://doi.org/10.1109/TWC.2015.2411651

8. C.-T. Lin, W.-R. Wu, C.-Y. Liu, Low-Complexity ML Detectors for generalized spatial modulation systems. IEEE Trans. Commun. 63(11), 4214-4230 (2015). https://doi.org/10.1109/TCOMM.2015.2469781

9. M.D. Renzo, H. Haas, A. Ghrayeb, S. Sugiura, L. Hanzo, in Proc. of the IEEE. Spatial modulation for generalized MIMO: challenges, opportunities, and implementation (2014), pp. 56-103. https://doi.org/10.1109/JPROC.2013.2287851

10. H.G. Myung, L. Junsung, D. Goodman, Single carrier FDMA for uplink wireless transmission. IEEE Veh. Technol. Mag. 1(3), 30-38 (2006). https://doi. org/10.1109/MVT.2006.307304

11. LTE, Evolved Universal Terrestrial Radio Access (E-UTRA); Physical channels and modulation, ETSI TS 136 211, V14.3.0., (2017).

12. A. Silva, J. Assunção Assunção, R. Dinis, A. Gameiro, Performance evaluation of IB-DFE-based strategies for SC-FDMA systems. EURASIP J. Wirel. Commun Netw. 2013, 292 (2013). https://doi.org/10.1186/1687-1499-2013-292

13. D. Castanheira, A. Silva, R. Dinis, A. Gameiro, Efficient transmitter and receiver designs for SC-FDMA based heterogeneous networks. IEEE Trans. Commun 63(7), 2500-2510 (2015). https://doi.org/10.1109/TCOMM.2015.2434383

14. S. Kamal, C.A.A. Meza, N.H. Tran, K. Lee, Low-PAPR hybrid filter for SC-FDMA. IEEE Commun. Lett. 21(4), 905-908 (2017). https://doi.org/10.1109/LCOMM. 2016.2617310

15. K. Wu, G. Ren, M. Yu, PAPR reduction of SC-FDMA signals using optimized additive pre-distortion. IEEE Commun. Lett. 19(8), 1446-1449 (2015). https:// doi.org/10.1109/LCOMM.2015.2443092

16. D. Sinanović, G. Šišul, B. Modlic, Low PAPR Spatial modulation for SC-FDMA. IEEE Trans. Veh. Technol. 66(9), 443-454 (2017). https://doi.org/10.1109/TVT. 2016.2548002

17. M.F. Naeiny, F. Marvasti, Selected mapping algorithm for PAPR reduction of space-frequency coded OFDM systems without side information. IEEE Trans. Veh. Technol. 60(3), 1211-1216 (2011). https://doi.org/10.1109/TVT.2011. 2109070

18. C. Ni, T. Jiang, W. Peng, Joint PAPR reduction and sidelobe suppression using signal cancelation in NC-OFDM-based cognitive radio systems. IEEE Trans. Veh. Technol 64(3), 964-972 (2015). https://doi.org/10.1109/TVT.2014. 2327012

19. J. Ji, G. Ren, H. Zhang, PAPR Reduction in Coded SC-FDMA Systems via Introducing Few Bit Errors. IEEE Commun. Lett. 18(7), 1258-1261 (2014). https://doi.org/10.1109/LCOMM.2014.2327964

20. P. Yang, Y.L. Guan, Y. Xiao, M.D. Renzo, S. Li, L. Hanzo, Transmit precoded spatial modulation: maximizing the minimum euclidean distance versus minimizing the bit error ratio. IEEE Trans. Wireless Commun. 15(3), 20542068 (2016). https://doi.org/10.1109/TWC.2015.2497692

21. P.W. Wolniansky, G.J. Foschini, G.D. Golden, R.A. Valenzuela, in Conference Proceedings. V-BLAST: An architecture for realizing very high data rates over the rich-scattering wireless channel. in Proc. of URSI International Symposium on Signals, Systems, and Electronics (1998), pp. 295-300. https://doi.org/10. 1109/ISSSE.1998.738086

22. G.J. Foschini, G.D. Golden, R.A. Valenzuela, P.W. Wolniansky, Simplified processing for high spectral efficiency wireless communication employing 
multi-element arrays. IEEE J. Sel. Areas Commun. 17(11), 1841-1852 (1999). https://doi.org/10.1109/49.806815

23. M. Debbah, B. Muquet, M. De Courville, M. Muck, S. Simoens, P. Loubaton, in A MMSE successive interference cancellation scheme for a new adjustable hybrid spread OFDM system. in Proc. of VTC2000-Spring IEEE 51st Vehicular Technology Conference (2000), pp. 745-749. https://doi.org/10.1109/NETECS.2000.851224

24. R. Bohnke, D. Wubben, V. Kuhn, K.D. Kammeyer, in IEEE Global Telecommunications Conference. Reduced complexity MMSE detection for BLAST architectures. Proc. of GLOBECOM '03 (2003), pp. 2258-2262. https:// doi.org/10.1109/GLOCOM.2003.1258637

25. T. Jiang, Y. Wu, An Overview: Peak-to-average power ratio reduction techniques for OFDM signals. IEEE Trans. on Broadcast 54(2), 257-268 (2008). https://doi.org/10.1109/TBC.2008.915770

26. G. Berardinelli, C.N. Manchon, L. Deneire, T.B. Sorensen, P. Mogensen, K. Pajukoski, in Turbo Receivers for Single User MIMO LTE-A Uplink. Proc. of VTC Spring 2009 - IEEE 69th Vehicular Technology Conference (2009), pp. 1-5. https://doi.org/10.1109/VETECS.2009.5073893

27. B. Dhivagar, K. Kiran, G. Krishnamurthy, An iterative DFE receiver for MIMO SC-FDMA uplink. IEEE Commun. Lett. 18(12), 2141-2144 (2014). https://doi org/10.1109/LCOMM.2014.2361787

28. M. Jiang, P. Narayan, W. Xiaodong, in Design of high performance MIMO receivers for LTE/LTE-A uplink. in Proc. of 2010 Conference Record of the Forty Fourth Asilomar Conference on Signals, Systems and Computers (2010), pp. 1-5. https://doi.org/10.1109/ACSSC.2010.5757785

29. LTE, Evolved Universal Terrestrial Radio Access (E-UTRA); User equipment (UE) radio transmission and reception, ETSI TS 136 101, V14.3.0., 2017.

30. P. Bao, Q. Guan, M. Guan, A multiuser detection algorithm in the uplink SCFDMA system for green communication network. IEEE Access 4(2016), 5982-5989 (2016). https://doi.org/10.1109/ACCESS.2016.2556279

31. F.E. Abd El-Samie, F.S. Al-kamali, A.Y. Al-nahari, M.I. Dessouky, SC-FDMA for mobile communications (Taylor \& Francis group, Boca Raton, 2014), pp. 1540. https://doi.org/10.1201/b15157

32. H. Holma, A. Toskala, LTE for UMTS-OFDMA and SC-FDMA based radio access (Wiley, Chichester, 2009), pp. 67-82. https://doi.org/10.1002/9780470745489

\section{Publisher's Note}

Springer Nature remains neutral with regard to jurisdictional claims in published maps and institutional affiliations.

\section{Submit your manuscript to a SpringerOpen ${ }^{\circ}$ journal and benefit from:}

- Convenient online submission

- Rigorous peer review

- Open access: articles freely available online

High visibility within the field

- Retaining the copyright to your article

Submit your next manuscript at $\boldsymbol{\nabla}$ springeropen.com 